\title{
A note on some expansions of $p$-adic functions
}

\author{
by
}

Grzegorz Szkibiel (Szczecin)

Introduction. Recently J. Rutkowski (see [3]) has defined the $p$-adic analogue of the Walsh system, which we shall denote by $\left(\phi_{m}\right)_{m \in \mathbb{N}_{0}}$. The system $\left(\phi_{m}\right)_{m \in \mathbb{N}_{0}}$ is defined in the space $C\left(\mathbb{Z}_{p}, \mathbb{C}_{p}\right)$ of $\mathbb{C}_{p}$-valued continuous functions on $\mathbb{Z}_{p}$. J. Rutkowski has also considered some questions concerning expansions of functions from $C\left(\mathbb{Z}_{p}, \mathbb{C}_{p}\right)$ with respect to $\left(\phi_{m}\right)_{m \in \mathbb{N}_{0}}$.

This paper is a remark to Rutkowski's paper. We define another system $\left(h_{n}\right)_{n \in \mathbb{N}_{0}}$ in $C\left(\mathbb{Z}_{p}, \mathbb{C}_{p}\right)$, investigate its properties and compare it to the system defined by Rutkowski. The system $\left(h_{n}\right)_{n \in \mathbb{N}_{0}}$ can be viewed as a $p$-adic analogue of the well-known Haar system of real functions (see [1]). It turns out that in general functions are expanded much easier with respect to $\left(h_{n}\right)_{n \in \mathbb{N}_{0}}$ than to $\left(\phi_{m}\right)_{m \in \mathbb{N}_{0}}$. Moreover, a function in $C\left(\mathbb{Z}_{p}, \mathbb{C}_{p}\right)$ has an expansion with respect to $\left(h_{n}\right)_{n \in \mathbb{N}_{0}}$ if it has an expansion with respect to $\left(\phi_{m}\right)_{m \in \mathbb{N}_{0}}$. At the end of this paper an example is given of a function which has an expansion with respect to $\left(h_{n}\right)_{n \in \mathbb{N}_{0}}$ but not with respect to $\left(\phi_{m}\right)_{m \in \mathbb{N}_{0}}$.

Throughout the paper the ring of $p$-adic integers, the field of $p$-adic numbers and the completion of its algebraic closure will be denoted by $\mathbb{Z}_{p}, \mathbb{Q}_{p}$ and $\mathbb{C}_{p}$ respectively ( $p$ prime). In addition, we write $\mathbb{N}_{0}=\mathbb{N} \cup\{0\}$ and $E=\{0,1, \ldots, p-1\}$.

The author would like to thank Jerzy Rutkowski for fruitful comments and remarks that permitted an improvement of the presentation.

Definition and basic properties. Let $p$ be a fixed prime number and $n \in \mathbb{N}_{0}$. If $n \neq 0$ then for some $k \in \mathbb{N}_{0}$ we have $n=n_{0}+n_{1} p+\ldots+n_{k} p^{k}$, where $n_{i} \in E$ for $i \in\{0,1, \ldots, k\}$ and $n_{k} \neq 0$. Define $n_{-}=n_{0}+n_{1} p+\ldots+$ $n_{k-1} p^{k-1}, n_{+}=n_{k}, n_{p}=p^{k}$. Let $\zeta$ be a primitive $p$-root of unity in $\mathbb{C}_{p}$. The functions $h_{0}, h_{1}, \ldots$ are defined as follows: $h_{0} \equiv 1$ and for $n>0$ we put

$$
h_{n}(x):= \begin{cases}n_{p} \zeta^{n_{+} x_{k}} & \text { if } x \in n_{-}+n_{p} \mathbb{Z}_{p} \\ 0 & \text { otherwise }\end{cases}
$$


where $x=x_{0}+x_{1} p+\ldots+x_{k} p^{k}+\ldots$ is a $p$-adic integer number in Hensel's form (i.e. $x_{i} \in E$ ).

Before proving some properties of $\left(h_{n}\right)_{n \in \mathbb{N}_{0}}$, we shall introduce some notation. For $f \in C\left(\mathbb{Z}_{p}, \mathbb{C}_{p}\right)$ define

$$
\bar{f}(x):= \begin{cases}f(x)^{-1} & \text { if } f(x) \neq 0 \\ 0 & \text { otherwise. }\end{cases}
$$

The function $\langle\cdot, \cdot\rangle: C\left(\mathbb{Z}_{p}, \mathbb{C}_{p}\right) \times C\left(\mathbb{Z}_{p}, \mathbb{C}_{p}\right) \rightarrow \mathbb{C}_{p}$ defined by

$$
\langle f, g\rangle:=\int_{\mathbb{Z}_{p}} f \bar{g}=\lim _{k \rightarrow \infty} p^{-k} \sum_{j=0}^{p^{k}-1} f(j) \bar{g}(j)
$$

has some properties of the inner product. We shall see that the system $\left(h_{n}\right)_{n \in \mathbb{N}_{0}}$ is orthogonal with respect to the above defined "inner product". Moreover, we define

$$
V_{k}:=\left\{f \in C\left(\mathbb{Z}_{p}, \mathbb{C}_{p}\right): \forall x, y \in \mathbb{Z}_{p}\left(x \equiv y\left(\bmod p^{k}\right) \Rightarrow f(x)=f(y)\right)\right\} .
$$

Observe that $V_{k}$ is a $p^{k}$-dimensional vector space over $\mathbb{C}_{p}$. Now we shall prove

Theorem 1. Let $x=x_{0}+x_{1} p+\ldots+x_{k} p^{k}+\ldots \in \mathbb{Z}_{p}\left(x_{i} \in E\right)$. The functions $h_{0}, h_{1}, \ldots$ have the following properties:

(a) $\left|h_{0}(x)\right|_{p}=1, \quad\left|h_{n}(x)\right|_{p}= \begin{cases}n_{p}^{-1} & \text { if } x \in n_{-}+n_{p} \mathbb{Z}_{p}, \\ 0 & \text { otherwise, }\end{cases}$ where $|\cdot|_{p}$ denotes the $p$-adic norm;

(b) $\sum_{j=0}^{p^{k}-1} h_{n_{+} n_{p}+j}(x)=n_{p} \zeta^{n_{+} x_{k}}$;

(c) $h_{n}$ is continuous for all $n \in \mathbb{N}_{0}$;

(d) $\left\langle h_{n}, h_{m}\right\rangle= \begin{cases}n_{p}^{-1} & \text { if } n=m, \\ 0 & \text { otherwise; }\end{cases}$

(e) $h_{0}, h_{1}, \ldots, h_{p^{k}-1}$ form a basis in the vector space $V_{k}$ over $\mathbb{C}_{p}$.

Proof. Properties (a)-(c) are easy to verify. Let $n=n_{0}+n_{1} p+\ldots+$ $n_{r} p^{r}, m=m_{0}+m_{1} p+\ldots+m_{s} p^{s}$ and $j=j_{0}+j_{1} p+\ldots+j_{k-1} p^{k-1}$, where $n_{r} \neq 0, m_{s} \neq 0$ and all coefficients are in $E$. To prove (d) consider the following sum for $k>\max \{s, r\}$ :

$$
S=\sum_{j=0}^{p^{k}-1} h_{n}(j) \bar{h}_{m}(j) .
$$

Assume $r>s$. Then

$$
h_{n}(j) \bar{h}_{m}(j) \neq 0 \quad \text { iff } \quad j \equiv n\left(\bmod p^{r}\right) \text { and } m \equiv n\left(\bmod p^{s+1}\right),
$$


So

$$
\begin{aligned}
S= & \sum_{j_{r}=0}^{p-1} \sum_{i=0}^{p^{k-r-1}-1} h_{n}\left(j_{r} p^{r}+i p^{r+1}\right) \\
& \quad \times \bar{h}_{m}\left(n_{s} p^{s}+n_{s+1} p^{s+1}+\ldots+n_{r-1} p^{r-1}+j_{r} p^{r}+i p^{r+1}\right) \\
= & p^{r-s} \zeta^{-m_{s} n_{s}} \sum_{i=0}^{p^{k-r-1}-1}\left(\sum_{j_{r}=0}^{p-1} \zeta^{n_{r} j_{r}}\right)=0 .
\end{aligned}
$$

Reasoning similarly for $r<s$ one also obtains $S=0$. If $r=s$ then

$$
h_{n}(j) \bar{h}_{m}(j) \neq 0 \quad \text { iff } \quad j \equiv n\left(\bmod p^{r}\right) \text { and } m \equiv n\left(\bmod p^{r}\right) .
$$

If $n_{r} \neq m_{r}$ then

$$
\begin{aligned}
S & =\sum_{j_{r}=0}^{p-1} \sum_{i=0}^{p^{k-r-1}-1} h_{n}\left(j_{r} p^{r}+i p^{r+1}\right) \bar{h}_{m}\left(j_{r} p^{r}+i p^{r+1}\right) \\
& =p^{k-r-1} \sum_{j_{r}=0}^{p-1} \zeta^{\left(n_{r}-m_{r}\right) j_{r}}=0 .
\end{aligned}
$$

Otherwise (i.e. when $n_{r}=m_{r}$ ) one obtains $S=p^{k-r}=p^{k} n_{p}^{-1}$. Therefore (d) holds.

(e) Observe that $h_{0}, h_{1}, \ldots, h_{p^{k}-1}$ belong to $V_{k}$. It now suffices to show that if $f \in V_{k}$ then

$$
\begin{aligned}
f= & \left(p^{-k} \sum_{j=0}^{p^{k}-1} f(j)\right) h_{0} \\
& +\sum_{n=1}^{p^{k}-1}\left(p^{-k} \sum_{j=0}^{p^{k-1} n_{p}^{-1}-1} \sum_{s=0}^{p-1} \zeta^{-n_{+} s} f\left(j p n_{p}+s n_{p}+n_{-}\right)\right) h_{n} .
\end{aligned}
$$

Denote the right side by $g$. It suffices to show that $f(r)=g(r)$ for $r \in$ $\left\{0,1, \ldots, p^{k}-1\right\}$, because for each $x \in \mathbb{Z}_{p}$ there exists $r \in\left\{0,1, \ldots, p^{k}-1\right\}$ such that $x \equiv r\left(\bmod p^{k}\right)$ and $f, g \in V_{k}$. Set

$$
S_{i}=\sum_{n=p^{i}}^{p^{k}-1} p^{-k} \sum_{j=0}^{p^{k-1} n_{p}^{-1}-1} \sum_{s=0}^{p-1} \zeta^{-n_{+} s} f\left(j p n_{p}+s n_{p}+n_{-}\right) h_{n}(r),
$$

where $i \in\{0,1, \ldots, k-1\}$.

Let $r=r_{0}+r_{1} p+\ldots+r_{k-1} p^{k-1}$, where $r_{0}, r_{1}, \ldots, r_{k-1} \in E$. Then 
one has

$$
\begin{aligned}
g(r) & =g\left(r_{0}+r_{1} p+\ldots+r_{k-1} p^{k-1}\right) \\
= & p^{-k} \sum_{s=0}^{p-1} \sum_{j=0}^{p^{k-1}-1} f(j p+s) \\
& +\sum_{n=1}^{p-1} p^{-k} \sum_{j=0}^{p^{k-1}} \sum_{s=0}^{p-1} \zeta^{-n s} f(j p+s) h_{n}\left(r_{0}+r_{1} p+\ldots+r_{k-1} p^{k-1}\right)+S_{1} \\
= & p^{-k} \sum_{n=0}^{p-1} \sum_{s=0}^{p-1} \sum_{j=0}^{p^{k-1}-1} \zeta^{n\left(r_{0}-s\right)} f(j p+s)+S_{1} .
\end{aligned}
$$

Observe that $\sum_{n=0}^{p-1} \zeta^{n\left(r_{0}-s\right)} \neq 0$ iff $s=r_{0}$, therefore

$$
g(r)=p^{-k} \sum_{j=0}^{p^{k-1}-1} p f\left(j p+r_{0}\right)+S_{1} .
$$

Reasoning in the same way one obtains

$$
\begin{aligned}
& g(r)= p^{-k} \sum_{j=0}^{p-1} p^{k-1} f\left(j p^{k-1}+r_{k-2} p^{k-2}+\ldots+r_{1} p+r_{0}\right)+S_{k-1} \\
&= p^{-k} \sum_{s=0}^{p-1} p^{k-1} f\left(s p^{k-1}+r_{k-2} p^{k-2}+\ldots+r_{1} p+r_{0}\right) \\
&+\sum_{n=p^{k-1}}^{p^{k}-1} p^{-k} \sum_{j=0}^{p^{0}-1} \sum_{s=0}^{p-1} \zeta^{-n_{+} s} f\left(j p^{k}+s p^{k-1}+n_{-}\right) h_{n}\left(r_{0}+r_{1} p+\ldots\right. \\
&\left.\ldots+r_{k-2} p^{k-2}+r_{k-1} p^{k-1}\right) .
\end{aligned}
$$

But if $r_{0}+r_{1} p+\ldots+r_{k-2} p^{k-2} \neq n_{-}$then $h_{n}(r)=0$ so one gets

$$
g(r)=p^{-k} \sum_{n_{+}=0}^{p-1} \sum_{s=0}^{p-1} p^{k-1} \zeta^{n_{+}\left(r_{k-1}-s\right)} f\left(s p^{k-1}+r_{k-2} p^{k-2}+\ldots+r_{1} p+r_{0}\right) \text {. }
$$

If $r_{k-1} \neq s$ then $\sum_{n_{+}=0}^{p-1} \zeta^{n_{+}\left(r_{k-1}-s\right)}=0$ so finally one obtains

$$
g(r)=f\left(r_{k-1} p^{k-1}+r_{k-2} p^{k-2}+\ldots+r_{1} p+r_{0}\right)=f(r) .
$$

Expansion of functions with respect to the system $\left(h_{n}\right)_{n \in \mathbb{N}_{0}}$. We start with some notations. The sequence $\left(x^{(k)}\right)_{k \in \mathbb{N}}$ where $x^{(k)}=x_{0}+$ $x_{1} p+\ldots+x_{k-1} p^{k-1}$ is called the standard sequence of the element $x=$ $x_{0}+x_{1} p+\ldots \in \mathbb{Z}_{p}$. The sequence $\left(f^{(k)}\right)_{k \in \mathbb{N}}$ where $f^{(k)}(x)=f\left(x^{(k)}\right)$ is 
called the standard sequence of the function $f \in C\left(\mathbb{Z}_{p}, \mathbb{C}_{p}\right)$. It is easy to see that

$$
\lim _{k \rightarrow \infty} x^{(k)}=x, \quad \lim _{k \rightarrow \infty} f^{(k)}(x)=f(x)
$$

for all $x \in \mathbb{Z}_{p}$ and that $f^{(k)} \in V_{k}$. So one may apply the formula (1) to $f^{(k)}$ and obtain

$$
f^{(k)}=\sum_{n=0}^{p^{k}-1} f_{n}^{(k)} h_{n},
$$

where

$$
\begin{aligned}
f_{0}^{(k)} & =p^{-k} \sum_{j=0}^{p^{k}-1} f(j), \\
f_{n}^{(k)} & =p^{-k} \sum_{j=0}^{p^{k-1} n_{p}^{-1}} \sum_{s=0}^{p-1} \zeta^{-n_{+} s} f\left(j p n_{p}+s n_{p}+n_{-}\right) \quad \text { if } 0<n<p^{k} .
\end{aligned}
$$

(If $p^{k-1} n_{p}^{-1}-1<0$ then put $f_{n}^{(k)}=0$.)

Definition 1. A function $f \in C\left(\mathbb{Z}_{p}, \mathbb{C}_{p}\right)$ has an expansion with respect to the system $\left(h_{n}\right)_{n \in \mathbb{N}_{0}}$ if the following conditions are satisfied:

(E1) for any $n \in \mathbb{N}_{0}$ the limit $f_{n}:=\lim _{k \rightarrow \infty} f_{n}^{(k)}$ exists;

(E2) $\lim _{n \rightarrow \infty} n_{p} f_{n}=0$.

Observe that (E2) implies the convergence of $\sum_{n=0}^{\infty} f_{n} h_{n}$. This series is called the expansion of $f$ with respect to $\left(h_{n}\right)_{n \in \mathbb{N}_{0}}$. We write $f \sim \sum_{n=0}^{\infty} f_{n} h_{n}$.

Remark. The series $\sum_{n=0}^{\infty} f_{n} h_{n}$ is also convergent if the sequence $\left(\left|f_{n}\right|_{p}\right)_{n \in \mathbb{N}_{0}}$ is bounded. Indeed, if there exists $M \in \mathbb{R}$ such that for any $n \in \mathbb{N}_{0}$ we have $\left|f_{n}\right|_{p} \leq M$ then

$$
0 \leq\left|f_{n} n_{p}\right|_{p} \leq M\left|n_{p}\right|_{p} \quad \text { and } \quad \lim _{n \rightarrow \infty}\left|n_{p}\right|_{p}=0,
$$

so (E2) holds and the series $\sum_{n=0}^{\infty} f_{n} h_{n}$ is convergent.

The next theorem follows immediately from the above definition.

THEOREM 2. The set of all functions which have an expansion with respect to $\left(h_{n}\right)_{n \in \mathbb{N}_{0}}$ is a vector space over $\mathbb{C}_{p}$.

The following result describes a class of functions which have an expansion with respect to $\left(h_{n}\right)_{n \in \mathbb{N}_{0}}$. 
THeOREM 3. If there exist constants $d_{0}, d_{1}, \ldots \in \mathbb{C}_{p}$ such that $f=$ $\sum_{m=0}^{\infty} d_{m} h_{m}$ then $f$ has an expansion with respect to $\left(h_{n}\right)_{n \in \mathbb{N}_{0}}$ and $f \sim$ $\sum_{m=0}^{\infty} d_{m} h_{m}$.

Proof. It is sufficient to compute $f_{n}^{(k)}$ where $k, n \in \mathbb{N}_{0}$, and to show that $\lim _{k \rightarrow \infty} f_{n}^{(k)}=d_{n}$. For $n=0$ one has

$$
f_{0}^{(k)}=p^{-k} \sum_{j=0}^{p^{k}-1} d_{0}+\sum_{m=1}^{\infty} d_{m}\left(p^{-k} \sum_{j=0}^{p^{k}-1} h_{m}(j) \bar{h}_{0}(j)\right)=d_{0},
$$

by virtue of (2) and the proof of Theorem $1(\mathrm{~d})$. For $n>0$, consider the sum

$$
S=\sum_{j=0}^{p^{k-1} n_{p}^{-1}-1} \sum_{s=0}^{p-1} \zeta^{-n_{+} s} h_{m}\left(j p n_{p}+s n_{p}+n_{-}\right) .
$$

Using the definition of $\left(h_{n}\right)_{n \in \mathbb{N}_{0}}$ and the properties of roots of unity one obtains $S=0$ if $n \neq m$ and $S=p^{k}$ if $n=m$.

Finally, one has

$$
\begin{aligned}
f_{n}^{(k)}=\sum_{m \neq n} d_{m}\left(p^{-k} \sum_{j=0}^{p^{k-1}} \sum_{s=0}^{n_{p}^{-1}-1} \zeta^{p-1}{ }^{-n_{+} s} h_{m}\left(j p n_{p}+s n_{p}+n_{-}\right)\right) \\
+d_{n} p^{-k} \sum_{j=0}^{p^{k-1} n_{p}^{-1}} \sum_{s=0}^{p-1} \zeta^{-n_{+} s} h_{n}\left(j p n_{p}+s n_{p}+n_{-}\right)=d_{n} .
\end{aligned}
$$

Now one can see that $\lim _{k \rightarrow \infty} f_{n}^{(k)}=d_{n}$ for $n \in \mathbb{N}_{0}$, so (E1) holds. By convergence of $\sum_{m=0}^{\infty} d_{m} h_{m}$, (E2) also holds.

From the above theorem one can deduce the following two corollaries:

Corollary 4. If $f=\sum_{m=0}^{\infty} d_{m} h_{m}=\sum_{m=0}^{\infty} d_{m}^{\prime} h_{m}$ then $d_{m}=d_{m}^{\prime}$.

Corollary 5. If the expansions of $f, g \in C\left(\mathbb{Z}_{p}, \mathbb{C}_{p}\right)$ with respect to $\left(h_{n}\right)_{n \in \mathbb{N}_{0}}$ are convergent to those functions, then $f g$ has an expansion with respect to $\left(h_{n}\right)_{n \in \mathbb{N}_{0}}$.

Proof. Let $f=\sum_{n=0}^{\infty} f_{n} h_{n}$ and $g=\sum_{n=0}^{\infty} g_{n} h_{n}$. These series are absolutely convergent so their product is also absolutely convergent. Hence one may change the order of its terms. Because the product $h_{n} h_{m}$ is again $h_{s}$ or $\lambda h_{s}$ (for some $s \in \mathbb{N}_{0}, \lambda \in \mathbb{C}_{p}$ ) one can obtain the series $f g=\sum_{s=0}^{\infty} d_{s} h_{s}$ as a product $\left(\sum_{n=0}^{\infty} f_{n} h_{n}\right)\left(\sum_{n=0}^{\infty} g_{n} h_{n}\right)$. To finish the proof it is enough to apply Theorem 3 .

Now we shall give a few examples of expansions with respect to $\left(h_{n}\right)_{n \in \mathbb{N}_{0}}$. 
EXAMPles of EXPANSIONS. (a) Identity on $\mathbb{Z}_{p}(f(x)=x)$. Employing formulas (2) and Definition 1 one obtains

$$
\begin{aligned}
f_{0} & =\lim _{k \rightarrow \infty} p^{-k} \sum_{j=0}^{p^{k}-1} j=\lim _{k \rightarrow \infty}\left(p^{-k} 2^{-1}\left(p^{k}-1\right) p^{k}\right)=-2^{-1}, \\
f_{n} & =\lim _{k \rightarrow \infty} p^{-k} \sum_{j=0}^{p^{k-1} n_{p}^{-1}} \sum_{s=0}^{p-1} \zeta^{-n_{+} s}\left(j p n_{p}+s n_{p}+n_{-}\right) \\
& =p^{-1} \sum_{s=0}^{p-1} s \zeta^{-n_{+} s} \text { for } n>0 .
\end{aligned}
$$

Note that if $p=2$ one gets $f_{n}=-2^{-1}$ for all $n \in \mathbb{N}_{0}$.

(b) Quadratic function $\left(f(x)=x^{2}\right)$. It follows by direct computation that

$$
\begin{aligned}
f_{0} & =\lim _{k \rightarrow \infty} p^{-k} \sum_{j=0}^{p^{k}-1} j^{2}=\lim _{k \rightarrow \infty}\left(p^{-k} 6^{-1}\left(p^{k}-1\right) p^{k}\left(2 p^{k}-1\right)\right)=6^{-1}, \\
f_{n} & =\lim _{k \rightarrow \infty} p^{-k} \sum_{j=0}^{p^{k-1} n_{p}^{-1}} \sum_{s=0}^{p-1} \zeta^{-n_{+} s}\left(j p n_{p}+s n_{p}+n_{-}\right)^{2} \\
& =\lim _{k \rightarrow \infty} \sum_{s=0}^{p-1} s \zeta^{-n_{+} s}\left(p^{-1} n_{p} s+p^{-1} n_{-}+n_{p}\left(p^{k-1}-1\right)\right) \\
& =\sum_{s=0}^{p-1} s \zeta^{-n_{+} s}\left(p^{-1} n_{p} s+p^{-1} n_{-}-n_{p}\right) \quad \text { for } n>0 .
\end{aligned}
$$

(c) Exponential function $\left(f(x)=\exp (a x)\right.$ where $\left.|a|_{p}<p^{1 /(p-1)}\right)$. In this case, using the properties of the function exp, one gets

$$
\begin{aligned}
& f_{0}= \lim _{k \rightarrow \infty} p^{-k} \sum_{j=0}^{p^{k}-1} \exp (a j)=a(\exp (a)-1)^{-1}, \\
& f_{n}=\lim _{k \rightarrow \infty}\left(p^{-k} \sum_{j=0}^{p^{k-1} n_{p}^{-1}} \sum_{s=0}^{p-1} \zeta^{-n_{+} s} \exp \left(a j p n_{p}\right) \exp \left(a s n_{p}\right) \exp \left(a n_{-}\right)\right) \\
&=\lim _{k \rightarrow \infty}\left(p^{-k} \exp \left(a n_{-}\right)\left(\exp \left(a p^{k}\right)-1\right)\left(\exp \left(a p n_{p}\right)-1\right)^{-1}\right. \\
&\left.\times \sum_{s=0}^{p-1} \zeta^{-n_{+} s} \exp \left(a s n_{p}\right)\right)
\end{aligned}
$$


$=a \exp \left(a n_{-}\right)\left(\exp \left(a p n_{p}\right)-1\right)^{-1} \sum_{s=0}^{p-1} \zeta^{-n_{+} s} \exp \left(a s n_{p}\right) \quad$ for $n>0$.

(d) Trigonometric functions $(f(x)=\sin (a x), g(x)=\cos (a x)$, where $\left.|a|_{p}<p^{1 /(p-1)}\right)$. Applying well-known formulas one obtains

$$
\begin{aligned}
f_{0}= & \lim _{k \rightarrow \infty} p^{-k} \sum_{j=0}^{p^{k}-1} \sin (a j) \\
= & \lim _{k \rightarrow \infty}\left(p^{-k}\left(\sin \left(2^{-1} a\right)\right)^{-1} \sin \left(2^{-1} a\left(p^{k}-1\right)\right) \sin \left(2^{-1} a p^{k}\right)\right)=-2^{-1} a \\
f_{n}= & \lim _{k \rightarrow \infty} p^{-k} \sum_{j=0}^{p^{k-1} n_{p}^{-1}-1} \sum_{s=0}^{p-1} \zeta^{-n_{+} s} \sin \left(a j p n_{p}+a s n_{p}+a n_{-}\right) \\
= & \lim _{k \rightarrow \infty}\left(p^{-k}\left(\sin \left(2^{-1} a p n_{p}\right)\right)^{-1} \sin \left(2^{-1} a p n_{p}\left(p^{k-1} n_{p}^{-1}-1\right)\right)\right. \\
& +p^{-k}\left(\sin \left(2^{-1} a p n_{p}\right)\right)^{-1} \cos \left(2^{-1} a p n_{p}\left(p^{k-1} n_{p}^{-1}-1\right)\right) \\
= & \left.\times \sin \left(2^{-1} a p^{k}\right) \sum_{s=0}^{p-1} \zeta^{-n_{+} s} \sin \left(a s n_{p}+a n_{-}\right)\right) \\
& +\left(\tan \left(2^{-1} a p n_{p}\right)\right)^{-1} \sum_{s=0}^{p-1} \zeta^{-n_{+} s} \cos \left(a s n_{p}+a n_{-}\right)
\end{aligned}
$$

Reasoning in the same way one gets $g_{0}=2^{-1} a \cdot \tan \left(2^{-1} a\right)$ and

$$
\begin{aligned}
& g_{n}=2^{-1} a\left(\left(\tan \left(2^{-1} a p n_{p}\right)\right)^{-1} \sum_{s=0}^{p-1} \zeta^{-n_{+} s} \cos \left(a s n_{p}+a n_{-}\right)\right. \\
& \left.+\sum_{s=0}^{p-1} \zeta^{-n_{+} s} \sin \left(a s n_{p}+a n_{-}\right)\right) \quad \text { for } n>0 .
\end{aligned}
$$

(e) Characteristic function of a coset of the residue class field. Let $A=t+p^{r} \mathbb{Z}_{p}$, where $0 \leq t \leq p^{r-1}$. Then

$$
\chi_{A}(x)= \begin{cases}1, & x \in A \\ 0, & x \notin A\end{cases}
$$


Without any difficulty one obtains

$$
\left(\chi_{A}\right)_{0}=\lim _{k \rightarrow \infty} p^{-k} \sum_{j=0}^{p^{k}-1} \chi_{A}(j)=\lim _{k \rightarrow \infty} p^{-k} \sum_{i=0}^{p^{k-r}-1} \chi_{A}\left(t+i p^{r}\right)=p^{-r} .
$$

For $n>0$ assume $n=n_{0}+n_{1} p+\ldots+n_{a} p^{a}, t=t_{0}+t_{1} p+\ldots+t_{r-1} p^{r-1}$ and consider two cases $a<r$ and $a \geq r$. In the first case one gets

$$
\begin{aligned}
\left(\chi_{A}\right)_{n} & =\lim _{k \rightarrow \infty} p^{-k} \sum_{j=0}^{p^{k-a-1}} \sum_{s=0}^{-1} \zeta^{-n_{a} s} \chi_{A}\left(j p^{a+1}+s p^{a}+n_{-}\right) \\
& = \begin{cases}0 & \text { if } n_{-} \not \equiv t\left(\bmod p^{a}\right), \\
p^{-r} \zeta^{-n_{a} t_{a}} & \text { if } n_{-} \equiv t\left(\bmod p^{a}\right) .\end{cases}
\end{aligned}
$$

Considering the second case, note that if $n_{-} \not \equiv t\left(\bmod p^{r}\right)$ then $\left(\chi_{A}\right)_{n}=0$. Otherwise one obtains

$$
\begin{aligned}
\left(\chi_{A}\right)_{n} & =\lim _{k \rightarrow \infty} p^{-k} \sum_{j=0}^{p^{k-a-1}-1} \sum_{s=0}^{p-1} \zeta^{-n_{+} s} \chi_{A}\left(j p^{a+1}+s p^{a}+n_{-}\right) \\
& =p^{-a-1} \sum_{s=0}^{p-1} \zeta^{-n_{+} s}=0 .
\end{aligned}
$$

Relationship between $\left(h_{n}\right)_{n \in \mathbb{N}_{0}}$ and $\left(\phi_{m}\right)_{m \in \mathbb{N}_{0}}$. The aim of this section is to show that $f$ has an expansion with respect to $\left(h_{n}\right)_{n \in \mathbb{N}_{0}}$ if it has one with respect to the system $\left(\phi_{m}\right)_{m \in \mathbb{N}_{0}}$ defined by Rutkowski (see [3]), and to give an example of a function which has an expansion with respect to $\left(h_{n}\right)_{n \in \mathbb{N}_{0}}$ but not with respect to $\left(\phi_{m}\right)_{m \in \mathbb{N}_{0}}$. First recall the definition and basic properties of $\left(\phi_{m}\right)_{m \in \mathbb{N}_{0}}$. For $m=m_{0}+m_{1} p+\ldots+m_{s} p^{s} \in \mathbb{N}$ define

$$
\begin{aligned}
\phi_{m}(x) & =\phi_{m}\left(x_{0}+x_{1} p+\ldots+x_{s} p^{s}+\ldots\right)=\zeta^{x_{0} m_{0}+x_{1} m_{1}+\ldots+x_{s} m_{s}}, \\
\phi_{0}(x) & \equiv 1 .
\end{aligned}
$$

It follows immediately that

$$
\begin{aligned}
\phi_{m}\left(x_{0}+x_{1} p+\ldots+\right. & \left.x_{r} p^{r}+x_{r+1} p^{r+1}+\ldots\right) \\
& =\phi_{m}\left(x_{0}+x_{1} p+\ldots+x_{r} p^{r}\right) \phi_{m}\left(x_{r+1} p^{r+1}+\ldots\right) .
\end{aligned}
$$

The system $\left(\phi_{m}\right)_{m \in \mathbb{N}_{0}}$ is orthonormal in the sense of the definition given before Theorem 1 . The functions $\phi_{0}, \phi_{1}, \ldots, \phi_{p^{k}-1}$ form a basis in the vector space $V_{k}$ (see Theorem $1(\mathrm{e})$ ). For $f \in C\left(\mathbb{Z}_{p}, \mathbb{C}_{p}\right)$, elements of its standard 
sequence can be represented in the form

$$
f^{(k)}=\sum_{m=0}^{p^{k}-1}\left(p^{-k} \sum_{j=0}^{p^{k}-1} f(j) \bar{\phi}_{m}(j)\right) \phi_{m}, \quad \text { where } \bar{\phi}_{m}(j)=\phi_{m}(j)^{-1} .
$$

Define $\widehat{f}_{m}^{(k)}=p^{-k} \sum_{j=0}^{p^{k}-1} f(j) \bar{\phi}_{m}(j)$. Making use of the above notations we introduce

Definition 2. A function $f \in C\left(\mathbb{Z}_{p}, \mathbb{C}_{p}\right)$ has an expansion with respect to the system $\left(\phi_{m}\right)_{m \in \mathbb{N}_{0}}$ if the following holds:

(I) for any $m \in \mathbb{N}_{0}$ the limit $\widehat{f}_{m}=\lim _{k \rightarrow \infty} \widehat{f}_{m}^{(k)}$ exists;

(II) $\lim _{m \rightarrow \infty} \widehat{f}_{m}=0$.

Note that (II) guarantees the convergence of the series $\sum_{m=0}^{\infty} \widehat{f}_{m} \phi_{m}$, called the expansion of $f$ with respect to $\left(\phi_{m}\right)_{m \in \mathbb{N}_{0}}$. We write $f \sim \sum_{m=0}^{\infty} \widehat{f}_{m} \phi_{m}$. Now we prove the main theorem of this section.

THEOREM 6. A function $f$ has an expansion with respect to $\left(h_{n}\right)_{n \in \mathbb{N}_{0}}$ if it has one with respect to $\left(\phi_{m}\right)_{m \in \mathbb{N}_{0}}$.

Proof. First we transform the formulas for the coefficients $f_{n}^{(k)}$ in the expansion of $f$ with respect to $\left(h_{n}\right)_{n \in \mathbb{N}_{0}}$. For $n>0$ and $k$ large enough,

$$
\begin{aligned}
f_{n}^{(k)} & =p^{-k} \sum_{j=0}^{p^{k-1} n_{p}^{-1}-1} \sum_{s=0}^{p-1} \zeta^{-n_{+} s} f\left(j p n_{p}+s n_{p}+n_{-}\right) \\
& =p^{-k} \sum_{i=0}^{p^{k} n_{p}^{-1}-1} f\left(i n_{p}+n_{-}\right) \bar{h}_{n}\left(i n_{p}+n_{-}\right) n_{p} \\
& =n_{p} p^{-k} \sum_{i=0}^{p^{k} n_{p}^{-1}-1} \sum_{r=0}^{n_{p}-1} f\left(i n_{p}+r\right) \bar{h}_{n}\left(i n_{p}+r\right) \\
& =n_{p} p^{-k} \sum_{j=0}^{p^{k}-1} f(j) \bar{h}_{n}(j) .
\end{aligned}
$$

One can check that

$$
\bar{h}_{n}=n_{p}^{-2} \sum_{r=0}^{n_{p}-1} \bar{\phi}_{r}\left(n_{-}\right) \phi_{m_{+} n_{p}+r}
$$

$$
\text { (where } \left.-n_{+} \equiv m_{+}(\bmod p) \text { and } n>0\right) \text {, }
$$

$$
\bar{h}_{0} \equiv \bar{\phi}_{0}
$$


Applying (4) to (3) one obtains

$$
\begin{aligned}
f_{n}^{(k)} & =n_{p}^{-1} \sum_{r=0}^{n_{p}-1} \bar{\phi}_{r}\left(n_{-}\right) p^{-k} \sum_{j=0}^{p^{k}-1} f(j) \bar{\phi}_{n_{+} n_{p}+r}(j) \\
& =n_{p}^{-1} \sum_{r=0}^{n_{p}-1} \bar{\phi}_{r}\left(n_{-}\right) \widehat{f}_{n_{+} n_{p}+r}^{(k)} .
\end{aligned}
$$

The limit $f_{n}=\lim _{k \rightarrow \infty} f_{n}^{(k)}$ exists because $\widehat{f}_{n_{+} n_{p}+r}=\lim _{k \rightarrow \infty} \widehat{f}_{n_{+} n_{p}+r}^{(k)}$ exists by Definition 2 and $f_{n}=n_{p}^{-1} \sum_{r=0}^{n_{p}-1} \bar{\phi}_{r}\left(n_{-}\right) \widehat{f}_{n_{+} n_{p}+r}$, so condition (E1) of Definition 1 is satisfied. Now,

$$
\left|n_{p} f_{n}\right|_{p}=\left|\sum_{r=0}^{n_{p}-1} \bar{\phi}_{r}\left(n_{-}\right) \widehat{f}_{n_{+} n_{p}+r}\right|_{p} \leq \max \left\{\left|\widehat{f}_{n_{+} n_{p}+r}\right|_{p}: 0 \leq r \leq n_{p}\right\} .
$$

But $\lim _{n \rightarrow \infty} \widehat{f}_{n}=0$ so $\max \left\{\left|\widehat{f}_{n_{+} n_{p}+r}\right|_{p}: 0 \leq r \leq n_{p}\right\} \rightarrow 0$ as $n \rightarrow \infty$. Thus $\lim _{n \rightarrow \infty} n_{p} f_{n}=0$ and condition (E2) of Definition 1 is also satisfied.

Applying the above theorem and the result proved in [3], we immediately obtain the following

Corollary 7. (a) There exists a function $f \in C\left(\mathbb{Z}_{p}, \mathbb{C}_{p}\right)$ which has an expansion with respect to $\left(h_{n}\right)_{n \in \mathbb{N}_{0}}$ and $f \neq 0, f \sim 0$.

(b) Every uniformly differentiable function has an expansion with respect to $\left(h_{n}\right)_{n \in \mathbb{N}_{0}}$.

(c) There exists a differentiable function which does not have an expansion with respect to $\left(h_{n}\right)_{n \in \mathbb{N}_{0}}$.

Now we will show that the system $\left(h_{n}\right)_{n \in \mathbb{N}_{0}}$ is more general than $\left(\phi_{m}\right)_{m \in \mathbb{N}_{0}}$.

Example. Consider the function $f: \mathbb{Z}_{p} \rightarrow \mathbb{C}_{p}$ given by $f(0)=0$ and $f\left(x_{a} p^{a}+x_{a+1} p^{a+1}+\ldots\right)=p^{a+1} \zeta^{a+1}$, where $x_{a}$ is non-zero. One can check that $f$ is continuous. We shall show that $f$ has an expansion with respect to $\left(h_{n}\right)_{n \in \mathbb{N}_{0}}$, but the sequence $\left(\widehat{f}_{p^{s}}\right)_{s \in \mathbb{N}}$ is convergent to $2(p-1)$ so statement (II) of Definition 2 fails. We first prove the following facts:

(i) for $s \in \mathbb{N}, x \in \mathbb{Z}_{p}$ one has $f\left(p^{s} x\right)=p^{s} f(x)$;

(ii) $f\left(x_{a} p^{a}+x_{a+1} p^{a+1}+\ldots+x_{a+r} p^{a+r}+\ldots\right)=f\left(x_{a} p^{a}+x_{a+1} p^{a+1}+\right.$ $\left.\ldots+x_{a+r} p^{a+r}\right)$, where $x_{a}$ is non-zero and $r \geq 1$;

(iii) $\sum_{j=0}^{p^{k}-1} f\left(\alpha p^{s}+j p^{s+1}\right)=0$ for $s \in \mathbb{N}_{0}, k \in \mathbb{N}, \alpha \in E \backslash\{0\}$;

(iv) $\sum_{j=0}^{p^{k}-1} f(j)=(p-1) p^{k}$ for $k \in \mathbb{N}$.

The properties (i), (ii) are easy to verify and we get (iii) immediately by 
direct computations:

$$
\begin{aligned}
\sum_{j=0}^{p^{k}-1} f\left(\alpha p^{s}+j p^{s+1}\right) & =\sum_{i=0}^{p-1} \sum_{j=0}^{p^{k-1}-1} f\left(\alpha p^{s}+i p^{s+1}+j p^{s+2}\right) \\
& =p^{k-1} p^{s+1} \sum_{i=0}^{p-1} \zeta^{i}=0
\end{aligned}
$$

To prove (iv) write

$$
\begin{aligned}
\sum_{j=0}^{p^{k}-1} f(j)= & \sum_{j_{0}=1}^{p-1} \sum_{i=0}^{p^{k-1}-1} f\left(j_{0}+i p\right)+\sum_{j_{1}=1}^{p-1} \sum_{i=0}^{p^{k-1}-1} f\left(j_{1} p+i p^{2}\right)+\ldots \\
& +\sum_{j_{k-2}=1}^{p-1} \sum_{i=0}^{p^{k-1}-1} f\left(j_{k-2} p^{k-2}+i p^{k-1}\right)+\sum_{i=1}^{p-1} f\left(i p^{k-1}\right)+f(0) .
\end{aligned}
$$

The last two terms are 0 and $(p-1) p^{k}$ respectively by definition of $f$ while the others are zero by (iii).

Now we are ready to compute the coefficients $f_{n}$. Using (iv), (2) and Definition 1 , one obtains $f_{0}=p-1$. For $n>0$ we consider three cases: $1^{\mathrm{o}} n_{-}=0,2^{\mathrm{o}} n_{-}=a n_{p} p^{-1}$ (where $a \in E \backslash\{0\}$ ) and $3^{\circ} n_{-} \neq 0$ or $n_{-} \neq$ $a n_{p} p^{-1}$. In the first case one gets

$$
\begin{aligned}
f_{n} & =\lim _{k \rightarrow \infty} p^{-k} \sum_{j=0}^{p^{k-1} n_{p}^{-1}} \sum_{s=0}^{p-1} \zeta^{-n_{+} s} f\left(j p n_{p}+s n_{p}\right) \\
& =\lim _{k \rightarrow \infty} p^{-k} n_{p}\left(\sum_{s=1}^{p-1} \zeta^{-n_{+} s} \sum_{j=0}^{p^{k-1} n_{p}^{-1}-1} f(s+j p)+p \sum_{j=0}^{p^{k-1} n_{p}^{-1}-1} f(j)\right) .
\end{aligned}
$$

Here we have used (i). Applying (iii) and (iv) one can check that $f_{n}=p-1$. Consider the second case:

$$
\begin{aligned}
f_{n} & =\lim _{k \rightarrow \infty} p^{-k} \sum_{j=0}^{p^{k-1} n_{p}^{-1}-1} \sum_{s=0}^{p-1} \zeta^{-n_{+} s} f\left(j p n_{p}+s n_{p}+a n_{p} p^{-1}\right) \\
& =\lim _{k \rightarrow \infty} p^{-k} n_{p} p^{-1} p^{k-1} n_{p}^{-1} \sum_{s=0}^{p-1} p \zeta^{\left(1-n_{+}\right) s} .
\end{aligned}
$$

Here if $n_{+}=1$ then $f_{n}=1$ and otherwise $f_{n}=0$. Finally, if neither the first nor the second case holds then using (ii) one has 


$$
\begin{aligned}
f_{n} & =\lim _{k \rightarrow \infty} p^{-k} \sum_{j=0}^{p^{k-1} n_{p}^{-1}-1} \sum_{s=0}^{p-1} \zeta^{-n_{+} s} f\left(j p n_{p}+s n_{p}+n_{-}\right) \\
& =\lim _{k \rightarrow \infty} p^{-k} p^{k-1} n_{p}^{-1} f\left(n_{-}\right) \sum_{s=0}^{p-1} \zeta^{-n_{+} s}=0 .
\end{aligned}
$$

Since $f_{n} \in \mathbb{Z}_{p}$ for all $n \in \mathbb{N}_{0}$ the function $f$ has an expansion with respect to $\left(h_{n}\right)_{n \in \mathbb{N}_{0}}$ by the remark after Definition 1 .

Let us compute the coefficients $\widehat{f}_{p^{s}}($ where $s \in \mathbb{N})$ :

$$
\begin{aligned}
\widehat{f}_{p^{s}}= & \lim _{k \rightarrow \infty} p^{-k} \sum_{j=0}^{p^{k}-1} f(j) \bar{\phi}_{p^{s}}(j) \\
= & \lim _{k \rightarrow \infty} p^{-k} \\
& \times \sum_{i=0} \sum_{a=0}^{p^{s-1}-1} \sum_{b=0}^{p-1} \sum_{c=0}^{p-1} \sum_{j=0}^{p^{k-s-2}-1} f\left(i+a p^{s-1}+b p^{s}+c p^{s+1}+j p^{s+2}\right) \zeta^{-b} \\
= & \lim _{k \rightarrow \infty} p^{-k}\left(p^{k-s-1} \sum_{i=1}^{p^{s-1}-1} \sum_{a=0}^{p-1} f\left(i+a p^{s-1}\right) \sum_{b=0}^{p-1} \zeta^{-b}\right. \\
& +p^{k-s-1} \sum_{a=1}^{p-1} \sum_{b=0}^{p-1} f\left(a p^{s-1}+b p^{s}\right) \zeta^{-b} \\
& +p^{k-s-2} \sum_{b=1}^{p-1} \sum_{c=0}^{p-1} f\left(b p^{s}+c p^{s+1}\right) \zeta^{-b} \\
& \left.+\sum_{c=1}^{p-1} \sum_{j=0}^{k-s-2} f\left(c p^{s+1}+j p^{s+2}\right)+\sum_{j=0}^{p^{k-s-2}-1} f\left(j p^{s+2}\right)\right) .
\end{aligned}
$$

The first sum is zero. Applying (iii) one finds that the third and fourth sums are also zero. Using (i) and (iv) one shows that the fifth sum equals $p^{k}(p-1)$. Finally, applying the definition of $f$, one concludes that the second sum is $p^{k}(p-1)$. So $\widehat{f}_{p^{s}}=2(p-1)$.

\section{References}

[1] B. I. Golubov, A. V. Efimov and V. A. Skvortsov, Walsh Series and Walsh Transforms. Theory and Applications, Nauka, Moscow 1987, 9-41 (in Russian).

[2] N. Koblitz, p-adic Numbers, p-adic Analysis and Zeta-functions, Springer, New York 1977, 9-36, 91-117. 
[3] J. Rutkowski, On some expansions of p-adic functions, Acta Arith. 51 (1988), 233-345.

[4] W. H. Schikhof, Ultrametric Calculus, Cambridge University Press, 1984.

INSTITUTE OF MATHEMATICS

SZCZECIN UNIVERSITY

WIELKOPOLSKA 15

70-451 SZCZECIN, POLAND

Received on 21.5.1990

and in revised form on 4.7 .1991 